\title{
Some Problems on the Analysis of the Earth Tides
}

\author{
Takeshi MIKumo \\ Disaster Prevention Research Institute, Kyoto University \\ and \\ Ichiro NAKAGAWA \\ Geophysical Institute, Faculty of Science, Kyoto University
}

\begin{abstract}
The spectral structure of the earth tides which have been observed during one year by an Askania gravimeter at Kyoto, and that of the corresponding theoretical tides on a rigid earth, were compared to determine the tidal factor of gravity and the phase lag with a high precision. A band-pass filter that has dominant responses only for semidiurnal or diurnal frequencies was applied to both of the tidal functions, to eliminate the effects of drift and of contamination from other constituents. Variations in the two tidal parameters during the period were also examined, extracting only $M_{2}$ or $O_{1}$ wave by the use of a narrow Gaussian filter. These results show long-period fluctuations with several months, suggesting a limit for discussions on elastic and anelastic behavior of the earth.
\end{abstract}

\section{§1. Introduction}

For the analysis of the earth tides various methods have so far been developed, making significant contributions to the evaluation of overall elastic properties of the earth. It is noticed, however, that there have been rather scattered estimates for the tidal factor of gravity and the phase lag. The reason for this may be attributed partly to the effects of oceanic tides, atmospheric pressure and temperature variations or to local crustal structure, but it appears that some of the scattering arise from the methodological difference in the time length of analysis, the elimination of drift and the treatment of theoretical tides to be compared with observations.

Our main purpose in the present paper will be focused to determine, with a high precision, the tidal factor of gravity $G$ and particularly the phase lag $\kappa$ from more com plete analyses for reducing the above effects to a minimum extent, in order to test a future possibility of evaluating anelasticity of the whole earth from observations of the earth tides.

For this purpose, the following analyses are made by applying the Fourier transform and digital filtering techniques both to an observed record and to the corresponding theoretical tides. (1) The spectral structure of the observed tides and that predicted theoretically for a perfectly rigid earth are compared, and the two tidal parameters, $G$, and $\kappa$, for major constituents are then determined. (2) To eliminate the effects of drift and of contamination from other constituents, a band-pass filter which has dominant responses for semidiurnal or diurnal frequencies is applied to the observed and theoretical tides prior to the Fourier analysis. (3) A specific constituent $\left(M_{2}\right.$ or $\left.O_{1}\right)$ is extracted from the observed and theoretical tides using a narrow Gaussian filter, and the filtered functions are Fourier-analysed over successive time ranges with an appropriate length to examine possible time variations in $G$ and $\kappa$.

\section{§2. Observed and Theoretical Tides}

Data analysed are the earth tides record that has been obtained by the Askania gravimeter Gs-11 (No. 111) at the Geophysical Institute of Kyoto University $\left(35^{\circ} 02^{\prime} \mathrm{N}, 135^{\circ} 47^{\prime}\right.$ $\mathrm{E}, h=60 \mathrm{~m}$ ) during one year from August $1,00 \mathrm{~h} 00 \mathrm{~m}, 1959$ to July $31,23 \mathrm{~h} 00 \mathrm{~m}, 1960$ (NAKAGAWA, 1962). A total of 8,784 hourly 
values were digitized from the record.

In some conventional methods of tidal analysis, theoretical amplitudes of each tidal constituent are derived from the mean position of the tide-generating bodies, or with some corrections, but they do not correspond exactly to the amplitudes over a specific observation period. For a more appropriate comparison with observations, theoretical tidal variation of gravity based on a perfectly rigid earth has been computed here, for the same period and time intervals as the above observation, from some modification of LONGMAN's formulae (1959) referring to BARTEL'S (1957).

Both the observed and theoretical tides are then analysed by the same methods described below.

\section{§3. Spectral Structure}

The spectrum of the earth tides may be obtained by the conventional Fourier transform,

$$
F(\omega)=\frac{1}{T} \int_{-T}^{T} f(t) e^{-i \omega t} d t
$$

where $f(t)$ is the observed or theoretical tidal function and $2 T$ is the time interval of analysis. $F(\omega)$ is actually the spectrum of a truncated function, which is zero outside the interval $|t| \leqq T$. The truncation will cause contamination of the spectrum by energy leakage particularly in the vicinity of strong spectral peaks. Applying the Hanning data window

$$
\begin{aligned}
& D(t)=\frac{1}{2}\left(1+\cos \frac{\pi t}{T}\right) \text { for }|t| \leqq T \\
& =0 \quad \text { for }|t|>T
\end{aligned}
$$

to $f(t)$, to reduce the effects of contamination, we have

$$
\begin{aligned}
g(t) & =D(t) \cdot f(t) \\
& =\frac{1}{2} f(t)+\frac{1}{4} f(t) e^{i \omega_{0} t / 2}+\frac{1}{4} f(t) e^{-i \omega_{0} t / 2}
\end{aligned}
$$

Therefore,

$G(\omega)=\frac{1}{2} F(\omega)+\frac{1}{4} F\left(\omega+\frac{\omega_{0}}{2}\right)+\frac{1}{4} F\left(\omega-\frac{\omega_{0}}{2}\right)$

The spectrum $F(\omega)$ for the observed tides has been computed in a previous paper (NAKAGAWA, MIKumo and TANAKA, 1966) after removing a linear term of drift, so that the smoothed spectrum $G(\omega)$ can be obtained by the above procedure. The corresponding spectrum of the theoretical tides has also been computed here, using the same technique. Figs. 1 and 2 show the spectral structure of the observed and theoretical tides over semidiurnal and diurnal frequency ranges respectively. Although the application of the data window broadens the side lobe of each spectral peak and reduces its amplitude to about a half, the ordinate indicates the absolute amplitude, which has been corrected by multiplying a constant to recover the original amplitude for major constituents. The features of the observed amplitude spectrum are in close agreement with those of the theoretical spectrum, as have been demonstrated in some previous work (JOBERT, 1963a; BARSENKOV, 1967), except the absolute amplitudes, which are a function of the location of an observation station. It can be seen that spectral peaks occur for $M_{2}, S_{2}, N_{2}, K_{2}$ and $L_{2}$ in a semidiurnal frequency range and for $K_{1}, O_{1}, P_{1}, Q_{1}$ and $M_{1}$ in a diurnal range.

The tidal factor $G$ and the phase lag $\kappa$ were calculated from the amplitude and phase spectrums for the frequency appropriate to each constituent, as in the earlier work of this kind (HARRISON et al., 1963; SLICHTER et al., 1964),

$$
G=A_{0} / A_{T} \text { and } \kappa=\varphi_{O}-\varphi_{T}
$$

The results are given in the upper half of Table 1. The determined amplitudes and phases differ somewhat from those given in the previous paper, since the previous results have been corrected for the location of the moon and the sun to the central epoch of the observation.

\section{§4. Effects of the Drift and Other Tidal Constituents}

In the usual harmonic analysis, the instrumental drift involved in observed records had been eliminated by various methods such as Pertzev's or some other combinations of 

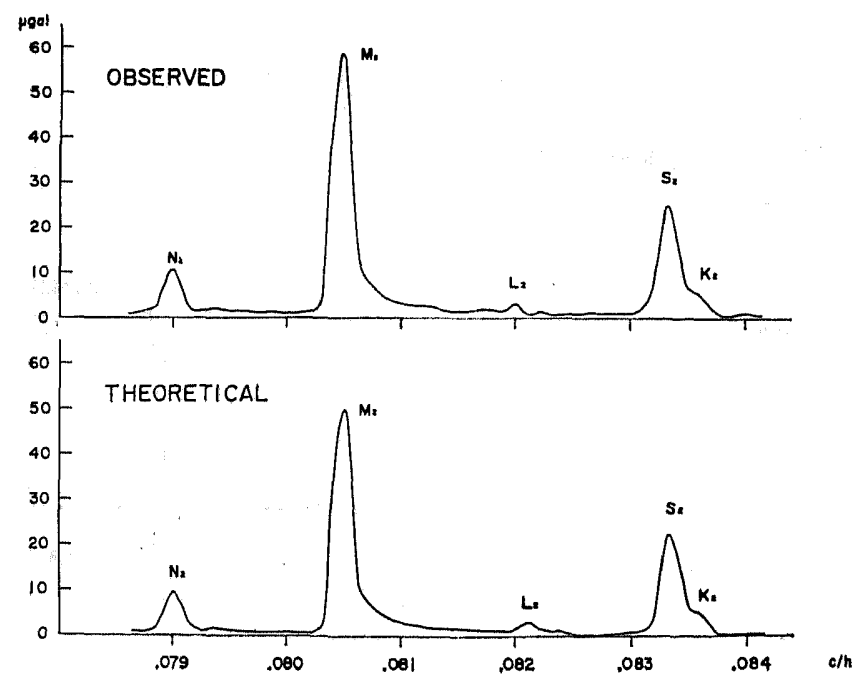

Fig. 1. Spectral structure over semidiurnal frequencies.

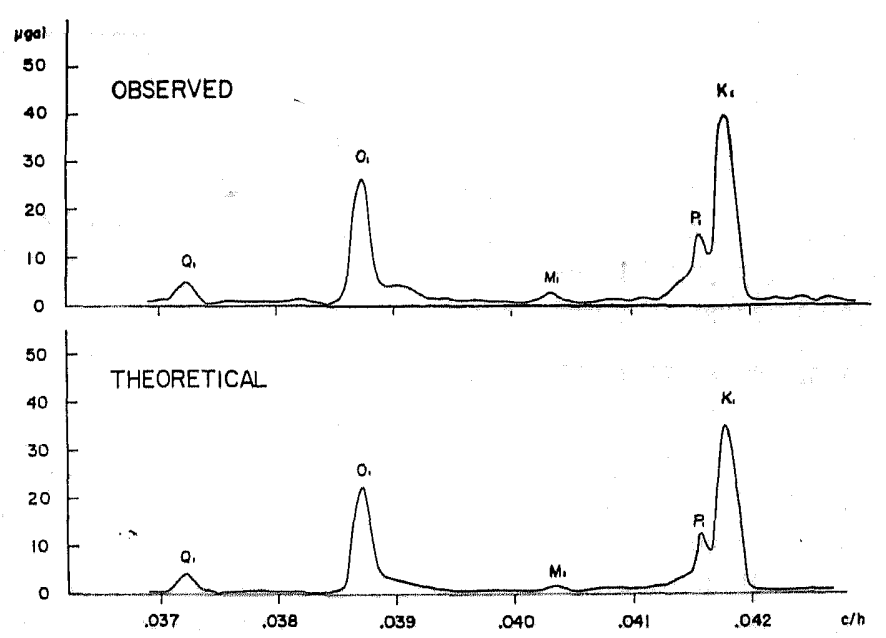

Fig. 2. Spectral structure over diurnal frequencies.

ordinates, but it does not appear that some of them have a sufficient precision for the present purpose.

An alternative technique is presented here to use a band-pass filter similar to that designed by JOBERT (1963b) and TANAKA and MIKUMo (1965), which has dominant responses only for semidiurnal or diurmal frequencies, rejecting all other frequencies as noises. Let the frequency response of the filter be $A(\omega)$ for $\omega_{1}<\omega<\omega_{2}$ and otherwise zero, then its impulse response $h(t)$ is,

$$
h(t)=\frac{1}{2 \pi} \int_{\omega_{1}}^{\omega_{2}} A(\omega) \cos \omega t d \omega
$$

which, if the interval $\left[\omega_{1}, \omega_{2}\right]$ is divided into $n$ subintervals $\left[\omega_{j}-\Delta \omega_{j} / 2, \omega_{j}+\Delta \omega_{j} / 2\right]$, may be approximated by,

$$
\begin{aligned}
\cong & \frac{1}{\pi} \sum_{j=1}^{n}\left[A\left(\omega_{j}\right) \Delta \omega_{j} \frac{\sin \varphi}{\varphi} \cos \omega_{j} t\right. \\
& \left.-\left(\frac{\partial A}{\partial \omega}\right)_{j} \Delta \omega_{j}\left(\frac{\sin \varphi}{\varphi}-\cos \varphi\right) \frac{\sin \omega_{j} t}{t}\right]
\end{aligned}
$$

where

$$
\varphi=\Delta \omega_{j} t / 2 .
$$

The filtered tides $\bar{f}(t)$ will be obtained by convolving the original tide function $f(t)$ with $h(t)$,

$$
\vec{f}(t)=\int_{-\tau_{1}}^{\tau_{1}} f(t-\tau) h(\tau) d \tau
$$

The limited length of the impulse response 
Table 1. Results of analysis.

$A_{O}$; amplitude of observed tides, $A_{r}$; amplitude of theoretical tides

$\varphi_{0}$; phase of observed tides, $\varphi_{T} ;$ phase of theoretical tides

$G$; tidal factor defined by $G=A_{O} / A_{T}$

$\kappa$; phase lag defined by $\kappa=\varphi_{O}-\varphi_{T}$

(1) results from unfiltered tides over the period:

August $1,00 \mathrm{~h} 00 \mathrm{~m}, 1959$-July $31,23 \mathrm{~h} 00 \mathrm{~m}$, 1960 (8784 hours)

(2) results from band-pass filtered tides over the period:

August $31,00 \mathrm{~h} 00 \mathrm{~m}, 1959$-June $25,23 \mathrm{~h} 00 \mathrm{~m}$, 1960 (7344 hours)

\begin{tabular}{|c|c|c|c|c|c|c|c|}
\hline & & \multicolumn{2}{|c|}{ Observed } & \multicolumn{2}{|c|}{ Theoretical } & \multirow{2}{*}{$G$} & \multirow{2}{*}{$\boldsymbol{\kappa}$} \\
\hline & & $A_{0}$ & $\varphi_{O}$ & $A_{T}$ & $\varphi_{T^{\prime}}$ & & \\
\hline \multirow{9}{*}{ (1) } & & $\mu \mathrm{gal}$ & deg. & $\mu$ gal & deg. & & deg. \\
\hline & $M_{2}$ & 60.535 & 170.02 & 52.243 & 172.00 & 1.160 & -1.98 \\
\hline & $S_{2}$ & 25.535 & 96.31 & 23.337 & 90.79 & 1.095 & 5.52 \\
\hline & $N_{2}$ & 10.504 & 312.49 & 9.874 & 315.89 & 1.064 & -3.40 \\
\hline & $K_{2}$ & 5.727 & 353.99 & 5.028 & 353.24 & 1.140 & 0.75 \\
\hline & $K_{1}$ & 40.992 & 171.99 & 36.152 & 173.87 & 1.134 & -1.88 \\
\hline & $O_{1}$ & 27.284 & 178.90 & 23.590 & 178.67 & 1.156 & 0.23 \\
\hline & $P_{1}$ & 15.136 & 94.47 & 13.532 & 95.38 & 1.120 & -0.91 \\
\hline & $Q_{1}$ & 5.644 & 320.34 & 4.477 & 321.81 & 1.260 & -1.47 \\
\hline \multirow{8}{*}{ (2) } & $M_{2}$ & 60.418 & 158.55 & 52.307 & 160.54 & 1.155 & -1.99 \\
\hline & $S_{2}$ & 26.179 & 97.48 & 23.843 & 92.03 & 1.098 & 5.45 \\
\hline & $N_{2}$ & 10.635 & 270.70 & 10.078 & 273.86 & 1.055 & -3.16 \\
\hline & $K_{2}$ & 7.677 & 25.62 & 6.689 & 21.41 & 1.147 & 4.21 \\
\hline & $K_{1}$ & 40.790 & 203.33 & 35.775 & 206.74 & 1.140 & -3.41 \\
\hline & $O_{1}$ & 27.355 & 138.26 & 23.561 & 137.84 & 1.161 & 0.42 \\
\hline & $P_{1}$ & 16.040 & 41.72 & 13.746 & 41.15 & 1.167 & 0.57 \\
\hline & $Q_{1}$ & 6.001 & 253.73 & 4.859 & 251.80 & 1.235 & 1.93 \\
\hline
\end{tabular}

gives a slight distortion to the designed filter, but a sufficiently long duration $\left(2 \tau_{1}=1440\right.$ hours) has reduced the distortion to a negligible extent, as may be seen in Figs. 3 and 4. The amplitude and phase of specific constituents filtered from the observed and theoretical tides were determined again by the Fourier transform in the same way as in equation (1), and the results obtained are shown in the lower half of Table 1. A comparison of the presented results with those from the unfiltered tides shows that the discrepancies in $G$ and $\kappa$ are less than $0.5 \%$ and $0.2^{\circ}$ respectively in both cases of $M_{2}$ and

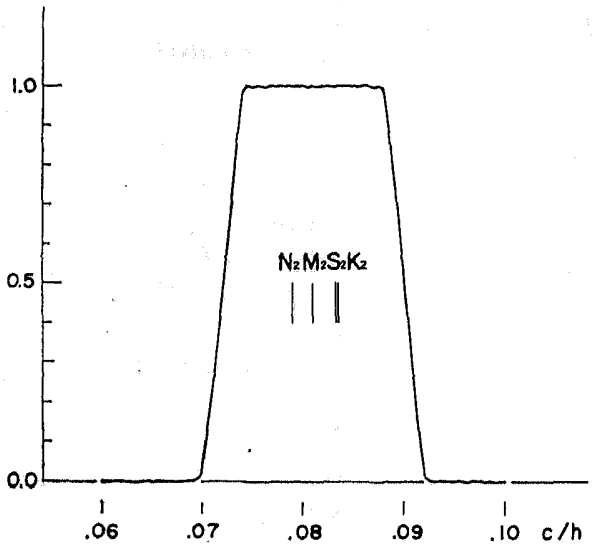

Fig. 3. Band-pass filter for semidiurnal frequencies.

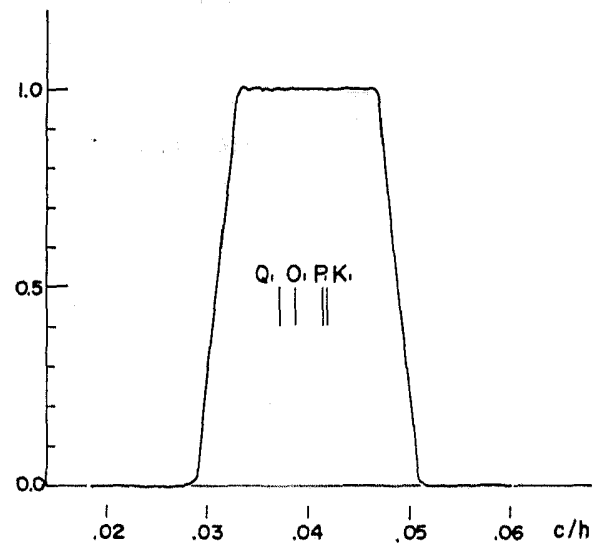

Fig. 4. Band-pass filter for diurnal frequencies.

$O_{1}$. The implication of the discrepancies is that, as far as we use the Fourier transform to determine the tidal parameters, the effects of the drift and of contamination from other constituents may be regarded as insignificant, as would be expected theoretically. The slight discrepancies may be due to shortening of the analysed time interval by two months in the filtered tides. The application of digital filtering of this kind may also be useful for the case with a more complex pattern of drift.

\section{$\S 5$. Variations in the Tidal Factor and the Phase Lag}

Another problem is to extract a specific constituent from the observed or theoretical tides by the use of a sharper filter like Gaus- 
sian's, for the purpose of examining whether significant variations in the tidal factor $G$ and the phase lag $\kappa$ exist during an observation period. The response of the Gaussian filter in the frequency domain is

$$
A(\omega)=e^{-\alpha\left(\omega-\omega_{c}\right)^{2}}
$$

Its impulse response is expressed as

$$
h(t)=\frac{1}{2 \sqrt{\pi \alpha}} \exp \left(-t^{2} / 4 \alpha\right) \cos \omega_{c} t
$$

where $\alpha$ is a constant which specifies a sharpness of the filter, and $\omega_{0}$ is the angular frequency which gives a maximum of $A(\omega)$. Figs. 5 and 6 show the Gaussian filter used

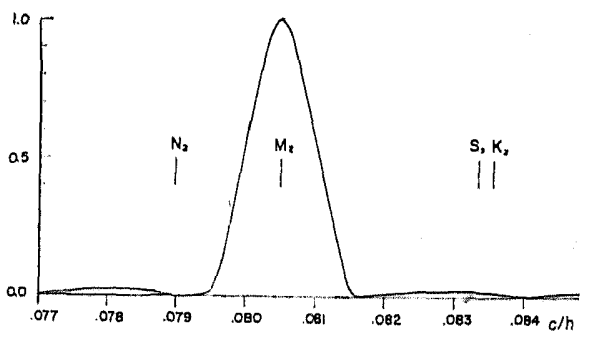

Fig. 5. Gaussian filter for $M_{2}$.

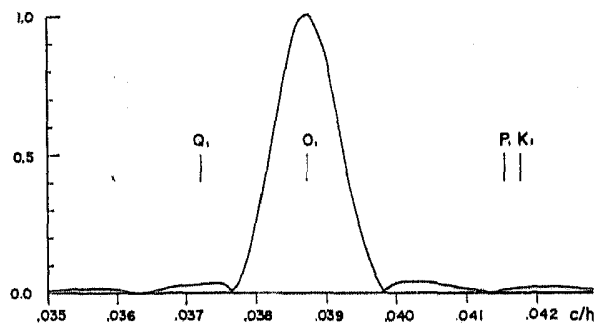

Fig. 6. Gaussian filter for $O_{1}$.

for $M_{2}$ and $O_{1}$ constituents, respectively. Small undulations at the wings result from a limited length of the impulse response. If the impulse response is convolved with the observed or theoretical tide function following equation (7), the filtered function $\bar{f}(t)$ consists mostly of a sinusoidal wave with an angular frequency of $\omega_{0}$. We tentatively divide the function over a length of $10 \mathrm{mon}$ ths into twenty time intervals, each having 15 days' period. The Fourier transform yields the amplitude and phase of $M_{2}$ or $O_{1}$ constituent for each of the divided intervals from the observed and theoretical tides res- pectively. In Table 2, thus determined values are tabulated. It is noted that the variation in the theoretical amplitudes over twenty intervals does not exceed $0.3 \%$ in the case of $M_{2}$ and $0.8 \%$ for $O_{1}$. This suggests that the amplitude of the constituent $M_{2}$ may be determined to a precision of about $0.3 \%$ from 15 days' filtered record and that time variations in the tidal factor exceeding the limit, if they exist, could be detected by the combined use of a sharp filter and the Fourier analysis.

The observed amplitudes indicate, however, variations as large as $2.5 \%$ in the case of $M_{2}$, and hence yield the same order of variation in $G$. The $O_{1}$ constituent shows much larger variations in the observed amplitudes and the tidal factor. The variations in $G$ and $\pi$ are shown in Figs. 7 and 8 . The vertical lines at the middle of each analysed interval indicate a possible range of the errors estimated from the accuracy of calibration for the gravimeter used. (NAKAGAWA, 1962). The tidal factor of $M_{2}$ is found to be between 1.13 and 1.18 and the corresponding phase lag ranges from $-0.6^{\circ}$ to $-3.8^{\circ}$. It may also be noticed from the two figures that there is a long-period fluctuation of about four months both in $G$ and $\kappa$, and that the fluctuation of $G$ is not in phase with that of $\kappa$.

\section{\$7. Discussion and Concluding Remarks}

Previous results (NAKAGAWA, 1962) for the same observation, which were obtained by Lecolazet's method, have yielded variations with higher frequencies in the two parameters both for $M_{2}$ and $O_{1}$. In some analyses using Lecolazet's or Pertzev's method, shorter-period fluctuations of $G$ with the order of several hours or several days have been described and interpreted as random observational errors (SARITSCEVA, 1964; KRAMER, 1964). It appears that long-period fluctuations that may be compared with the present case have been noted only in a long term observation (PARIISKII et al., 1967). It would be difficult, however, to attribute these fluctuations to any definite sources such as er- 


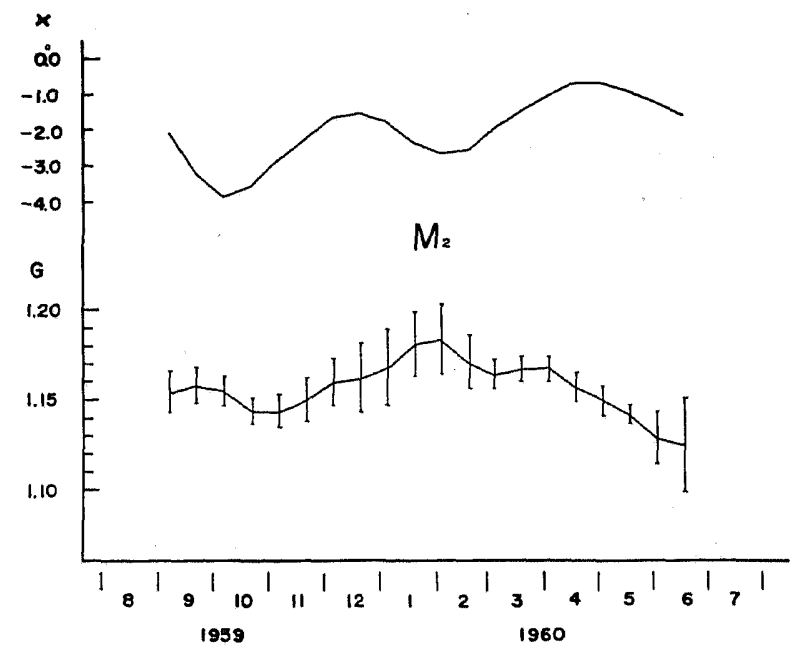

Fig. 7. Variations in $G$ and $\kappa$ of $M_{2}$ over every 15 days.

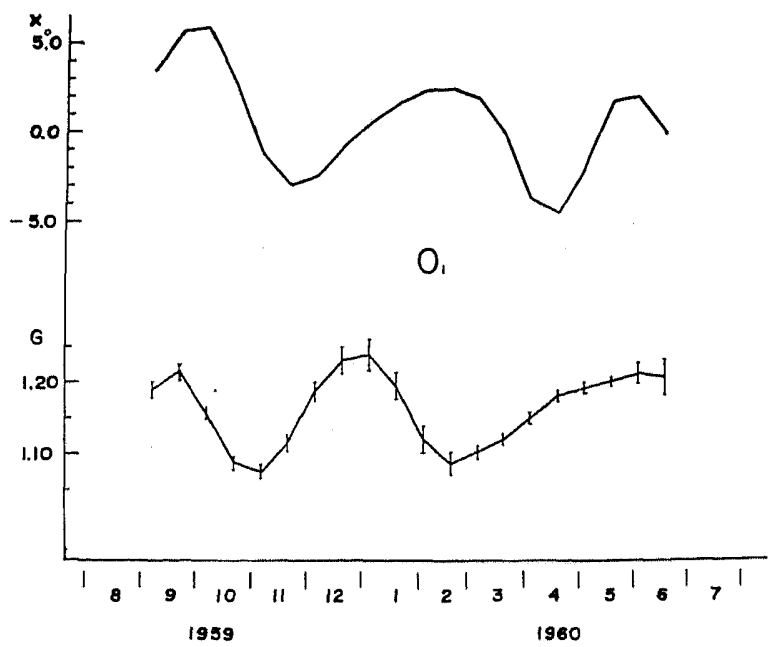

Fig. 8. Variations in $G$ and $\kappa$ of $Q_{1}$ over every 15 days.

rors in the instrumental calibration or drift, since the effects have been estimated or removed already.

To examine the effect of the time length of analysis and a possible correlation of the fluctuations with the amplitudes of the observed tides, similar analyses have been made of $M_{2}$ for four different time intervals; $a$ ) between two successive times for the full moon, $b$ ) the times for the new moon, $c$ ) the time from the full moon to the new moon, and $d$ ) between the time from the new moon to the full moon. The former two cases (a) and (b) correspond to a synodical month, which was taken to be 708 hours in the present analysis, while 354 hours were taken for the latter two cases (c) and (d). The tidal factor and the phase lag thus obtained are shown in Table 3 and plotted in Fig. 9 by different symbols. No significant differences can be recognized among the parameters determined for the four time intervals, and the variations in $G$ and $r$ agree also with the foregoing results (Fig. 7).

There leaves a possibility that seasonal variations in the effects of oceanic tides and 


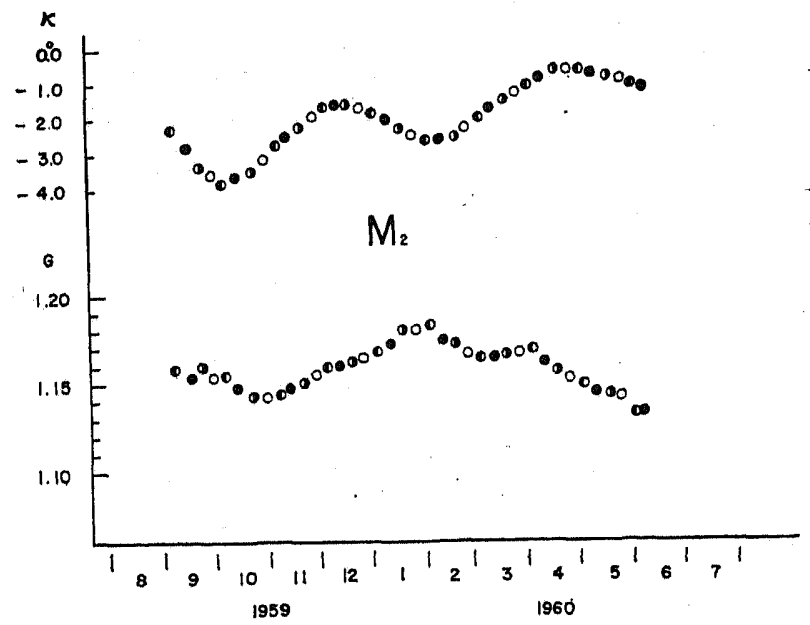

Fig. 9. Variations in $G$ and $\kappa$ of $M_{2}$ from the analysis for four different time intervals.

$O$; full moon-full moon ; new moon-new moon

; full moon-new moon ( new moon-full moon

Table 2. Variations in the tidal parameters of $M_{2}$ and $O_{1}$ over every 15 days' period.

\begin{tabular}{|c|c|c|c|c|c|c|c|c|c|c|c|c|c|}
\hline \multirow{2}{*}{ To. } & \multirow{2}{*}{ Period } & \multicolumn{6}{|c|}{$M_{2}$} & \multicolumn{6}{|c|}{$O_{1}$} \\
\hline & & $A_{0}$ & $\varphi_{0}$ & $A_{T}$ & $\varphi_{T}$ & $G$ & $n$ & $A_{o}$ & $\varphi_{0}$ & $A_{T}$ & $\varphi_{T}$ & $G$ & 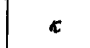 \\
\hline & & $\mu \mathrm{gal}$ & deg. & ugal & deg. & & deg. & $\mu \mathrm{gal}$ & deg. & $\mu$ gal & deg. & & deg. \\
\hline 1 & . 31-Sept. 14 & 60.374 & 158.47 & 52.315 & 160.58 & 1.154 & $\mid-2.11$ & 28.363 & 138.06 & 23.849 & 134.59 & 1.189 & 3.47 \\
\hline 2 & Sept. 15-Sept. 29 & 60.533 & 151.50 & 52.266 & 154.75 & 1.158 & -3.25 & 28.994 & 120.03 & 23.835 & 114.30 & 1.216 & 5.73 \\
\hline 3 & Sept. 30-Oct. 14 & 60.376 & 145.2 & 52.251 & 149.05 & 1.155 & -3.84 & 27.529 & 100.04 & 23.821 & 94.13 & 1.156 & 5.91 \\
\hline 4 & Oct. 15 -Oct. 29 & 59.693 & 139.66 & 52.199 & 143.23 & 1.144 & -3.57 & 25.785 & 76.71 & 23.753 & 73.87 & 1.086 & 2.84 \\
\hline 5 & Oct. 30 -Nov. 13 & 59.705 & 134.70 & 52.167 & 137.53 & 1.144 & -2.83 & 25.475 & 52.42 & 23.718 & 53.58 & 1.074 & -1.16 \\
\hline 6 & Nov. $14-$ Nov. 28 & 60.075 & 129 & 52.150 & 131.72 & 1.150 & -2.28 & 26.364 & 23.25 & 30.647 & 33.17 & 1.115 & -2.92 \\
\hline 7 & Nov. 29 -Dec. 13 & 60.470 & 124.3 & 52.135 & 126.02 & 1.160 & -1.70 & 28.103 & 10.35 & 23.676 & 12.83 & 1.187 & -2.48 \\
\hline 8 & Dec. 14-Dec. 28 & 60.640 & 118. & 52.180 & 120.22 & 1.162 & -1.57 & 29.174 & 351.62 & 23.710 & 352.35 & 1.230 & -0.73 \\
\hline 9 & Dec. 29-Jan. 12 & 60.960 & 112 & 52.201 & 114.50 & 1.168 & -1.80 & 29.470 & 332.81 & 23.781 & 332.15 & 1.239 & 0.66 \\
\hline 10 & $\begin{array}{l}1960 \\
\text { Jan. 13-Jan. } 27\end{array}$ & 01.700 & 10e & [12. & $\left.\right|^{2}+2$ & 1 & -2.32 & 200. & 313.62 & 20.091 & $\mid 311.86$ & 1.194 & 1.76 \\
\hline 11 & Jan. $28-F e b . ~ 11$ & 61.912 & 100 & 52.321 & 103.00 & 1.183 & -2.67 & 26.740 & 294.25 & 23.900 & 291.77 & 1.119 & 2.48 \\
\hline 12 & Feb. 12-Feb. 26 & 61.358 & 94.66 & 52.414 & 97.23 & 1.171 & -2.57 & 25.972 & 274.19 & 23.937 & 271.62 & 1.085 & 2.57 \\
\hline 13 & Feb. 27-Mar. 12 & 61.012 & 89.53 & 52.408 & 91.50 & 1.164 & $\mid-1.97$ & 26.269 & 253.44 & 23.859 & 251.40 & 1.101 & 2.04 \\
\hline 14 & Mar. 13-Mar. 27 & 61.218 & 84.26 & 62.463 & 85.76 & 1.167 & -1.50 & 26.661 & 230.18 & 23.820 & 231.06 & 1.119 & -0.88 \\
\hline 15 & Mar. 28-Apr. 11 & 61.211 & 78.99 & 52.401 & 80.02 & 1.168 & -1.03 & 27.313 & 206.05 & 23.747 & 7210.62 & 1.150 & -4.57 \\
\hline 16 & Apr. 12-Apr. 26 & 60.611 & 73.66 & 52.401 & 74.29 & 1.157 & -0.63 & 28.108 & 184.87 & 23.782 & 190.10 & 1.182 & -5.23 \\
\hline 17 & Apr. 27-May 11 & 60.148 & 67.89 & 52.305 & 68.54 & 1.150 & -0.65 & 28.406 & 167.86 & 23.790 & 169.63 & 1.194 & -1.77 \\
\hline 18 & May 12-May 26 & 59.676 & 61.98 & 52.278 & 62.82 & 1.142 & -0.84 & 28.760 & 151.35 & 23.916 & 149.33 & 1.203 & 2.02 \\
\hline 19 & May 27-June 10 & 58.934 & 55.93 & 52.193 & 57.05 & 1.129 & -1.12 & 29.091 & 131.43 & 23.969 & 129.05 & 1.214 & 2.38 \\
\hline 20 & June 11 -June 25 & 58.707 & 49.85 & 52.182 & 51.34 & 1.125 & -1.49 & & 109,35 & & 109.07 & 1.209 & 9.28 \\
\hline
\end{tabular}


in meteorological disturbances could contri- the phase of the fluctuation does not coincide bute to the long-period fluctuation of the between $M_{2}$ and $O_{1}$ and between $G$ and $\kappa$ tidal parameters. However, the result that introduces difficulty into the interpretation.

Table 3. Variations in the tidal parameters of $M_{2}$ over every 708 hours (a) from full moon to full moon, and from new moon to new moon, (b) and over every 354 hours from full moon to new moon, and from new moon to full moon.

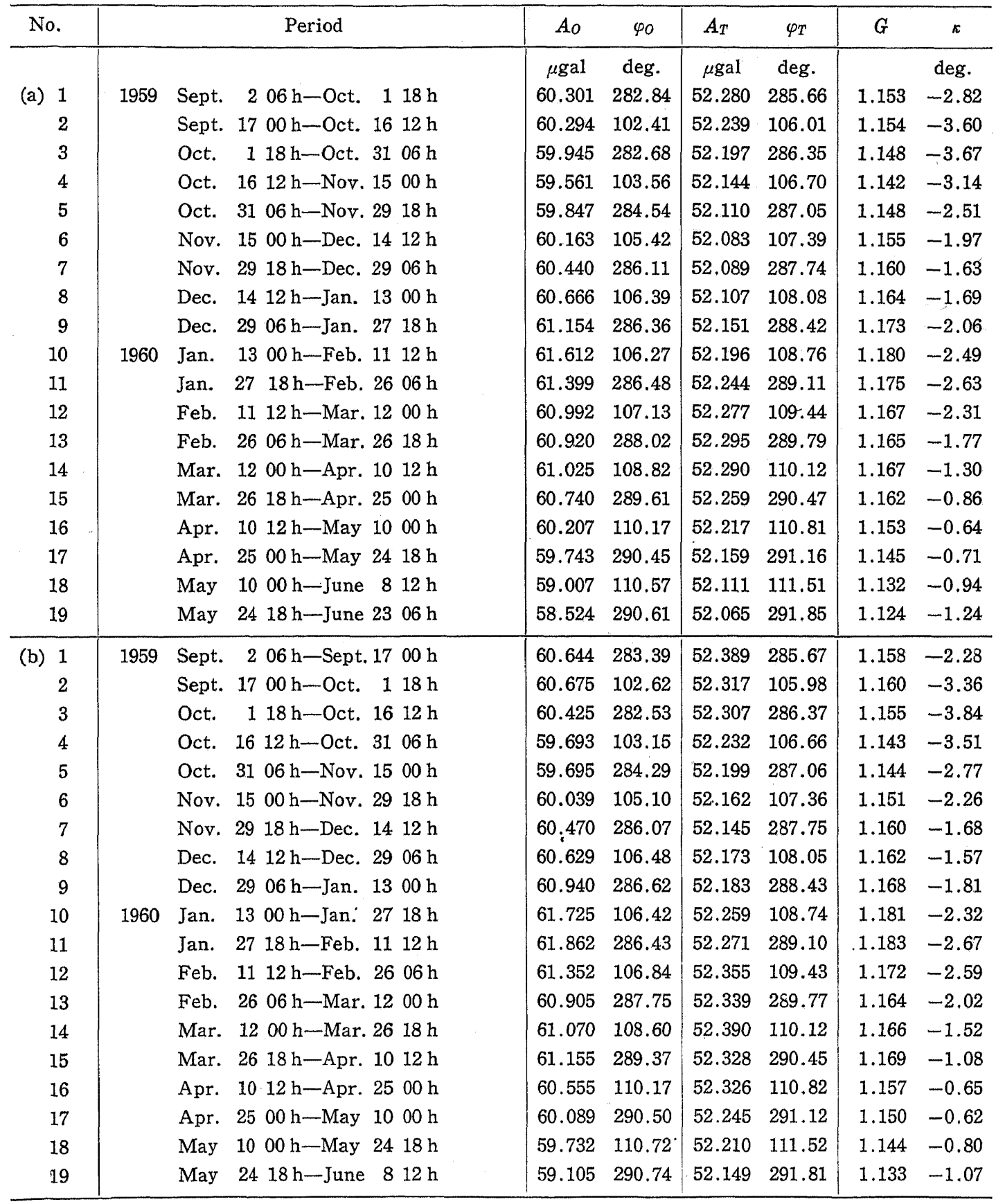


Detailed discussions on this problem require more observations covering a long period made concurrently at several stations with different kinds of gravimeters.

The rather large variations in the phase lag $\kappa$ make it difficult to evaluate anelasticity of the whole earth following MACDONALD's method (1964), although the best determination is that for $M_{2}$, a mean phase lag of which being about $-2^{\circ} .0$. Further developments on this side would not be expected, until the instrumental phase delay can be well calibrated and the effects of oceanic tides and other disturbances could be removed by proper techniques.

\section{Acknowledgments}

We wish to thank Drs. Michio Otsuka and Torao Tanaka for several comments on the manuscript, and Mrs. Ritsuko Koizumi for assistance in the analysis. Numerical computations were made on a HITAC 5020E at the Computation Center, University of Tokyo.

\section{References}

BARSENKOV, S. N.:

1967, A spectral analysis of tidal variations in the force of gravity at Talgar, Izv. Akad. Nauk, SSSR, 3 (English Translation), 170174.

BARTELS, J.:

1957, Gezeitenkräfte, Handbuch der Physik, XLVIII, Geophysik II, 747, Springer-Verlag, Berlin.

Harrison, J. C., N. F. Niss, I. M. Longman, R.F.S. Forbes, E. A. KraUt and L. B. SLICHTER:

1963, Earth-tide observations made during the International Geophysical Year, J. Geophys.

JOBERT, G.: Res., 68, 1497-1516.

1963a, Comparaison des résultas de l'analyse spectrale des marées terrestres avec les résultas théoriques, Marées Terrestres, Bulletin d'Informations, 33, 1013-1016.

JOBERT, G.:

1963b, Sur les filtres numeriques utilises dans l'analyse harmonique, ibid.,33, 1260-1273. Kramer, M. V.:

1964 , On the effect of chance errors of observations upon the results of determination of amplitudes and phases of elastic tidal waves, Communications Observatoire Royal de Belgique No. 236, Série Géophysique, 69, 426-437.

LONGMAN, I.M.:

1959, Formulas for computing the tidal accelerations due to the Moon and the Sun, J. Geophys. Res., 64, 2351-2355.

MacDonald, G. J.F.:

1964, Tidal friction, Rev. Geophys., 2, 524-530.

NAKAGAWA, I.:

1962, Some problems on time change of gravity, Part 3, On precise observation of gravity at the Gravity Reference Station, Bull. Disaster Prevention Res. Inst., Kyoto Univ., 57, 2-65.

NakagaWa, I., T. Mrkumo and T. Tanaka:

1966, Spectral structure of the earth tides and related phenomena-gravimetric record-, Spec. Contr. Geophys. Inst., Kyoto Univ., 6, 215-223.

Parisski, N. N., S. H. Barsenkov, V.A. Volkov, D. G. GridneV and M. V. Kramer:

1967, Results of 19 month observations of earth tides in Talgar, Izv. Akad. Nauk, SSSR, 10 (English Translation), 107-114.

SARITSCEVA, J. K.:

1964, Sur la dispersion des valeurs du facteur $\delta$ obtenue par la méthode de R. Lecolazet pour la meme station, Communications Observatoire Royal de Belgique No. 236, Série Géophysique, 69, 421-425.

Slichter, L.B., G. J.F. Macdonald, M. Caputo and C.L. HAGER:

1964, Report of earth tides results and of other gravity observations at UCLA, ibid., 172177.

Tanaka, T, and T. Mrkumo:

1965, An application of digital filtering to the record of ground deformation, J. Seism. Soc. Japan, Series II, 18, 235-244.

(Received July 25, 1968) 